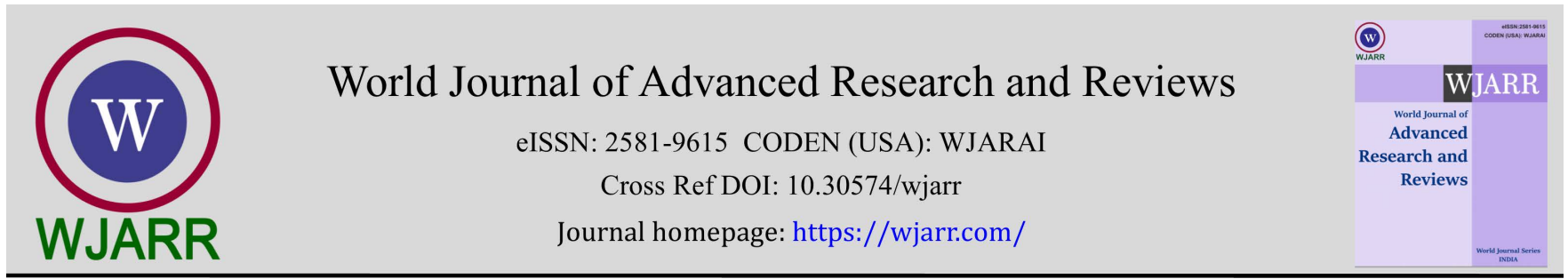

(RESEARCH ARTiCle)

Check for updates

\title{
Diagnoses of Neisseria Bacteria via Neural Networks Techniques
}

Kalthom Ibrahim 1, *, Mohammed Abdallah Almaleeh 2, Moaawia Mohamed Ahmed ${ }^{2}$ and Dalia Mahmoud Adam 1

${ }^{1}$ Faculty of Engineering, Department of Electrical Control, El neelain University, Khartoum, Sudan.

${ }^{2}$ Faculty of Computer and Information Technology, Department of Computer Engineering, Tabuk University, Tabuk, KSA.

World Journal of Advanced Research and Reviews, 2021, 12(03), 587-593

Publication history: Received on 06 November 2021; revised on 18 December 2021; accepted on 20 December 2021

Article DOI: https://doi.org/10.30574/wjarr.2021.12.3.0673

\begin{abstract}
This paper presented simple approach that automatically detects Neisseria Bacteria cell in the cerebrospinal fluid smear images. The proposed methodology mainly consists of cerebrospinal fluid smear images acquisition, transformation form red, green, blue smear images in to other color spaces. This step followed by subbing images and segmenting the images to extracting the images features then validation and classifying the Bacteria images based in features extracted using neural networks. The proposed diagnosis for Neisseria Bacteria through neural network techniques has performed high-precision performance in some suggested groups.
\end{abstract}

Keywords: Diseases; Neisseria bacteria cell; Cerebrospinal fluid; Classification; Diagnosis

\section{Introduction}

Meningitis is a health problem of the thin lining that surround the brain and spinal cord[1]. The type caused by bacteria is considered the most dangerous and can cause disabling, including brain injury and hearing loss, meningitis be diagnosed after death, due to the delay in diagnosis by the complex chemical and physical methods used to analyze patient samples. There are several types of meningitis infection[2]. It is a bacterial meningitis, a viral infection caused by viruses and parasitic fungi. The most important test in determining or excluding meningitis is the analysis of cerebrospinal fluid (CSF) through a lumbar puncture (LP, Spinal tap). CSF samples are checked for the presence of white blood cells, red blood cells, protein content and glucose concentration levels decreased is one of the most important indicators of bacterial meningitis [3].

Segmentation is the process in which an image is subdivided into regions or objects. These objects can be further processed or analyzed for the extraction of quantitative information[4]. Regularly combine segmentation is done with various morphological processing and filtering techniques to achieve an accurate and robust segmentation of an image[5]. Feature extraction is a leading to effectively differentiate various patterns. Various feature extraction in any classification system is the extraction of proper features [6]. Usually in feature extraction, the visual information of an image is analyzed in order to extract features such as shape, texture and color[7]. Regularly, initial processing of data set is done for two main reasons; Reduce the data size and data set for analysis that is more efficient and adapting the data set to better match the specific analysis method.

An artificial neural network (ANN) is an information processing system that attempts to mimic biological neural networks. In a neural network, the word network means the interconnection between neurons in different layers of a system. Each system is a three-layer system, an input layer, a hidden layer, and an output layer[8]. This paper summarizes taking pictures of cerebrospinal fluid infected and uninfected with Neisseria bacteria through optical

\footnotetext{
${ }^{*}$ Corresponding author: Kalthom Adam H. Ibrahim

Department of Electrical Control, Faculty of Engineering, El neelain University, Khartoum, Sudan.

Copyright (C) 2021 Author(s) retain the copyright of this article. This article is published under the terms of the Creative Commons Attribution Liscense 4.0.
} 
microscopy, then analyzing and classifying them with neural networks. This can serve as a diagnostic system for bacterial meningitis through computer-aided software. This paper aims to develop image processing and machine learning (ML) techniques to achieve higher accuracy in diagnosing Neisseria bacterial meningitis and optimal classification of meningococci[9].

\section{Related Work}

An estimated number of researches have conducted in the field of classification of biomedical images through computeraided programming. The new assessment of medical data mining tools has greatly influenced the healthcare industry, by improving the diagnosis of medical diseases and reducing the time pressure on clinicians. Magnetic resonance imaging (MRI) is widely used to classify biomedical images[10]. For example, the classification techniques for the detection of knee joint defects, which had an accuracy of 100\%[11]. The diagnosis of acute leukemia achieved an accuracy of $96 \%$ and $94 \%$ in extracting the blast cell region[12]. Applied research for diagnosing meningitis is based on chemical and physical methods, and there is rare research in this field that depends on the analysis program and electronic methods for diagnosing meningitis[13].

\section{Methodology}

The proposed method for approaching the diagnosis of Neisseria meningococcal disease is several stages of analysis; this involves preparing a smear of cerebrospinal fluid on a glass slide. The cerebrospinal fluid scan is treated with an appropriate Gram stain[14].

Then the samples encoded in a digital image for analysis and classification by software. The software used in this work is matlab R2014a. Cerebrospinal fluid samples were obtained from the microbiology laboratory of Mohamed El-Amin Hamid Children's Hospital, Omdurman, Sudan.

Color images of CSF smear first obtained as input to the diagnostic process, then processing the digital images with treatment programs to obtain clear images representing infection with bacterial parasites or images representing the natural components of the spinal fluid with its natural components without infected by Bacteria. Figure (1) illustrates the image of the normal CSF smear and figure (2) is infected one by Neisseria bacteria.

The images of the Neisseria bacteria analyzed and extracted their specifications and characteristics, these features are color, shape, and texture. There are 14 features extracted and evaluated. There are different techniques performed obtain good results depend on the region of interest (ROI) in the images[15]. In this work the process of segmentation including the isolating of the important region, by converting the image with red, green and blue (RGB) technology into a binary-color image, Then the edge of the image was used by the Canny's method, and the A mathematical operation process was applied such as filling and designing the structural element (disk) that leads to the extraction of the bacteria cells which isolated the area of interest from their internal components[16]. Neural networks have used as a classifier. Below is an input model for neural network coefficients for classification of Neisseria bacteria. Finally, the performance of all neural networks is evaluated[17].

- $\quad$ rnnet1=patternnet(20);

- $\quad$ rnnet.trainparam_epoches $=200$;

- rnnet.trainparam_lrs $=0.01$;

- rnnet.trainparam_goal=1e-3;

- rnnet.divideparam_trainRatio=70;

- $\quad$ rnnet.divideparam_valRatio=15;

- rnnet.divideparam_testRatio=15;

- rnnet.trainfcn='traing';

- rnnet.performFcn='mse' 


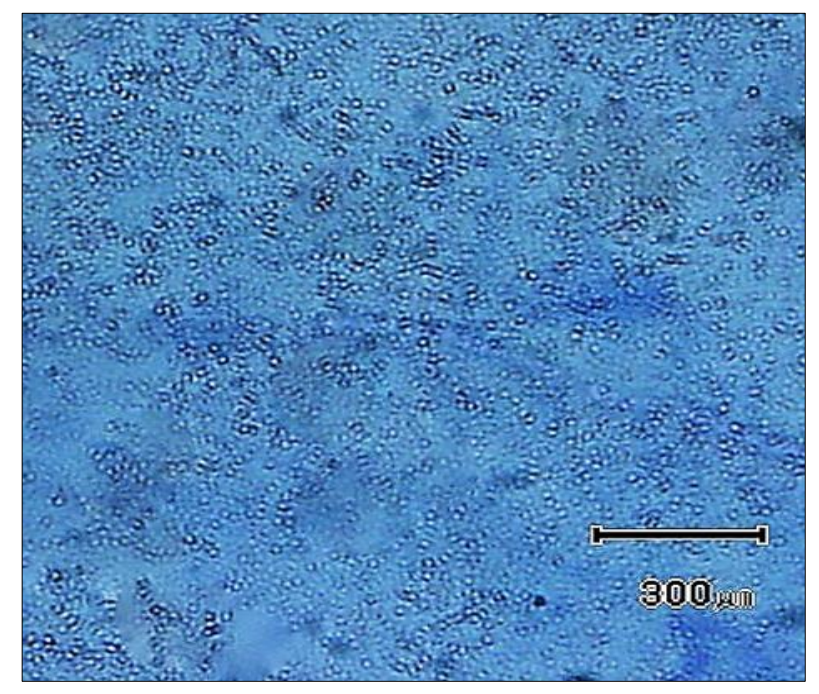

Figure 1 The image of normal CSF smear

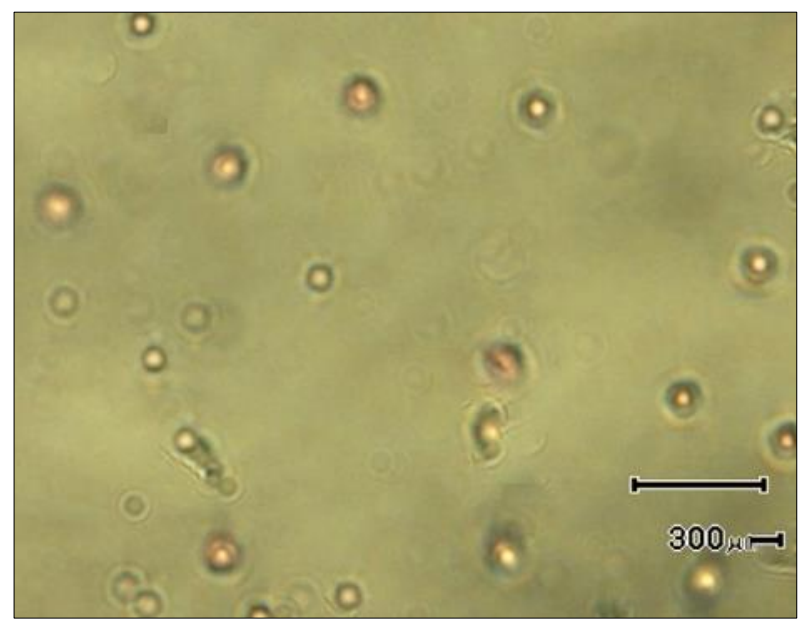

Figure 2 The image of CSF smear infected by Neisseria bacteria

\section{Results and discussion}

The use of relevant features to characterize an output class is essential for any classification problem. In this work, three types of features extracted from the Neisseria bacteria cells, namely shape, color, and texture. Six groups of features are considered, so the total amount of designed networks were six according to the features selected sets.

The performance of the network depending mainly in the feature set and the number of features which selected. Figure (3) illustrate one of the Neisseria network training performance and figure (4) shows the training performance frequency for the same Neisseria network[18].

According to the network accuracy graph in Figure (5) which started with the four characteristics that got the maximum network accuracy, all the parameters in this network are designed and tested until high accuracy is obtained. Then the slope incredibly drops to the lowest accuracy when the feature is increased to five, this increase in the number of features causes the accuracy and performance of the network to decrease, and the choice of the type of training parameters affected the performance of the network. The regression increases sharply when the features increase to nine, then there is a decrease by increasing the features to ten, then it is slightly stable when the features are increased to thirteen, and finally there is a slight increase by increasing the feature to fourteen[19]. 
It is noticed from this graph that there is small increase in the accuracy ,performance of the neural network directly proportional to the increase in the number and type of features extracted, However, it is clear that increasing the number of features in an unstudied manner leads to confusion in the neural network, and the selection of network parameters affects its performance[20].

Table (1) shows the extraction of image features and their corresponding network performance accuracy. However, it is in the Neisseria bacterium diagnostic system that acceptable accuracy obtained compared to performance results in previous research in biomedical image computer system diagnostics, such as the classification of acute leukemia that performs with an accuracy of $96 \%$ and $94 \%$ in blast cell region extraction[12].

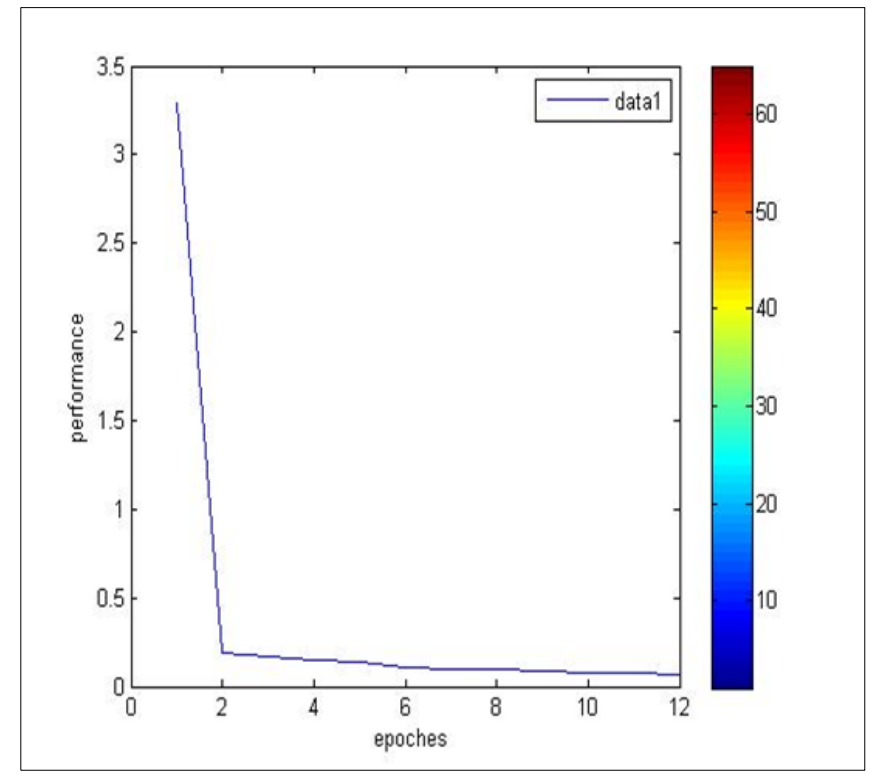

Figure 3 The network training performance for the Neisseria bacteria

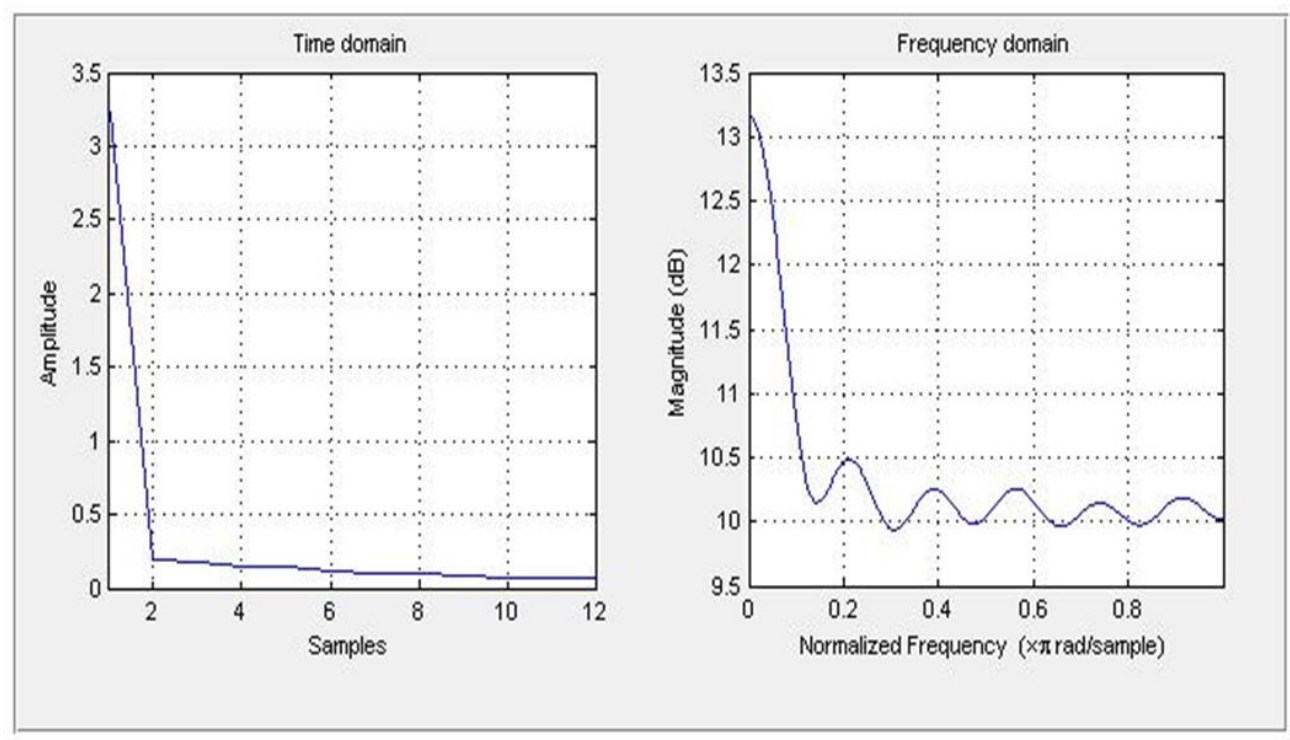

Figure 4 The Neisseria meningitidis training network performance frequency response 


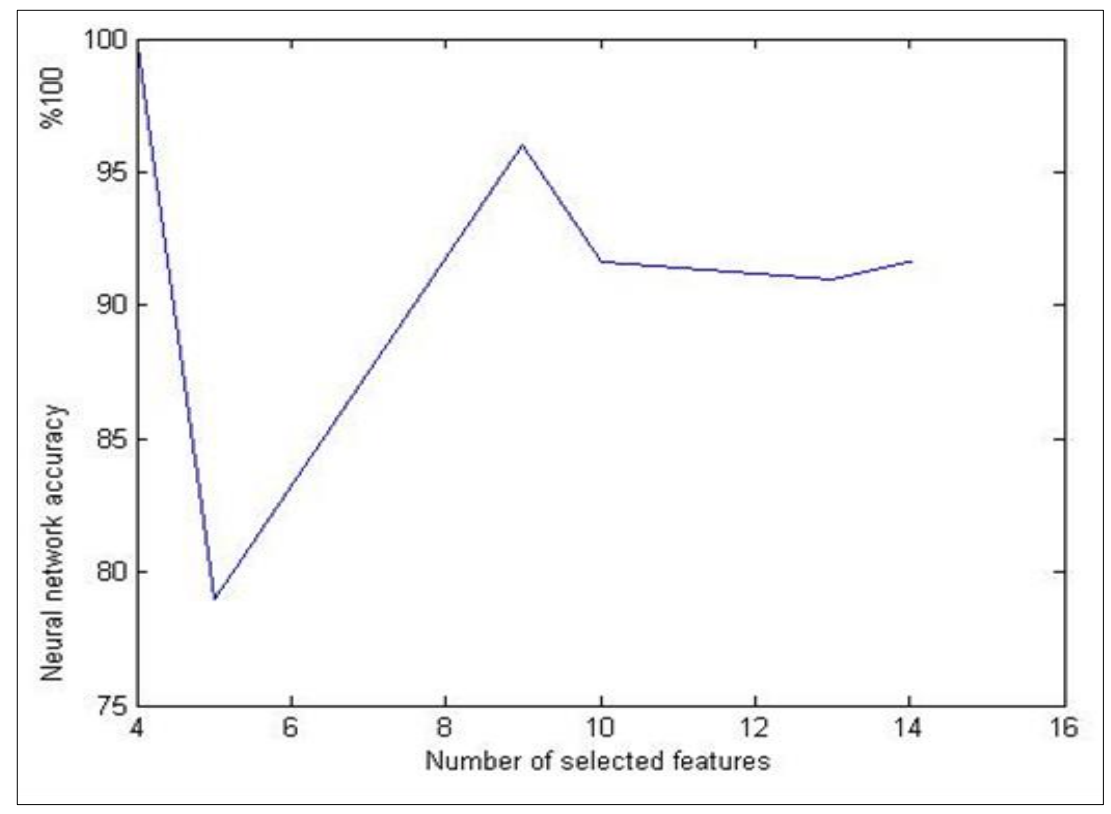

Figure 5 Neisseria bacteria classifying networks performance

Table 1 The correspondence results of Neisseria neural networks accuracy

\begin{tabular}{|c|c|}
\hline No of features & Accuracy (\%) \\
\hline 4 & 100.0000 \\
\hline 5 & 79.0000 \\
\hline 9 & 96.0000 \\
\hline 10 & 91.6600 \\
\hline 13 & 91.0000 \\
\hline 14 & 90.0000 \\
\hline
\end{tabular}

\section{Conclusion}

This work suggested a new methodology to design a system that divides and categorizes meningitis bacterial cells in CSF smear images. Control engineering tools and software were used to analyze medical problems to find new solutions for examining medical samples, which would save time for disease diagnosis in order to avoid human consequences of disease when diagnosis is delayed and sometimes death. The proposed methodology will achieve an acceptable segmentation and classification required to improve the system, when it is extensively tested in a larger number of CSF images under different conditions, so that thousands of images obtained by various staining methods and different cameras are required. Image segmentation can be applied through various types of segmentation, and features extracted from bacterial images can be categorized using different categorization techniques in order to design a highspeed, accurate and reliable system for diagnosing meningitis by computer-aided software[21].

\section{Compliance with ethical standards}

\section{Acknowledgments}

Authors are thankful for the research department of Khartoum state Health ministry and Al Amin Hamid children hospital, special thanks to laboratory staff for their continuous support in our research. The authors are grateful to Sara contactors and Al Adwa Alsara Company (Sharjah, U.A.E) for moral and material support. Special thanks expanded to those who contributed to the research with support, guidance, or care. 


\section{Disclosure of conflict of interest}

There is no conflict of interest in this study.

\section{Statement of ethical approval}

The human and social ethics followed in the research were adhered to

\section{References}

[1] J. Boulton, "Meninigitis immunisation: Challenges, successes and new developments," British Journal of Nursing, vol. 22, no. 1, pp. 20-25, 2013.

[2] R. Galimi, "Extrapulmonary tuberculosis: tuberculous meningitis new developments," Eur Rev Med Pharmacol Sci, vol. 15, no. 4, pp. 365-86, 2011.

[3] F. Deisenhammer et al., "Routine cerebrospinal fluid (CSF) analysis," European handbook of neurological management, vol. 1, pp. 5-17, 2011.

[4] A. S. Frangakis and R. Hegerl, "Segmentation of two-and three-dimensional data from electron microscopy using eigenvector analysis," Journal of Structural Biology, vol. 138, no. 1-2, pp. 105-113, 2002.

[5] F. Zana and J.-C. Klein, "Segmentation of vessel-like patterns using mathematical morphology and curvature evaluation," IEEE transactions on image processing, vol. 10, no. 7, pp. 1010-1019, 2001.

[6] G. G. Yen and K.-C. Lin, "Wavelet packet feature extraction for vibration monitoring," IEEE transactions on industrial electronics, vol. 47, no. 3, pp. 650-667, 2000.

[7] J. Yue, Z. Li, L. Liu, and Z. Fu, "Content-based image retrieval using color and texture fused features," Mathematical and Computer Modelling, vol. 54, no. 3-4, pp. 1121-1127, 2011.

[8] A. Thomas, "Memristor-based neural networks," Journal of Physics D: Applied Physics, vol. 46, no. 9, p. 093001, 2013.

[9] A. Delbaz et al., "Neisseria meningitidis induces pathology-associated cellular and molecular changes in trigeminal Schwann cells," Infection and immunity, vol. 88, no. 4, pp. e00955-19, 2020.

[10] A. K. Anaraki, M. Ayati, and F. Kazemi, "Magnetic resonance imaging-based brain tumor grades classification and grading via convolutional neural networks and genetic algorithms," biocybernetics and biomedical engineering, vol. 39, no. 1, pp. 63-74, 2019.

[11] C. D. Bocsa, V. Moisoiu, A. Stefancu, L. F. Leopold, N. Leopold, and D. Fodor, "Knee osteoarthritis grading by resonant Raman and surface-enhanced Raman scattering (SERS) analysis of synovial fluid," Nanomedicine: Nanotechnology, Biology and Medicine, vol. 20, p. 102012, 2019.

[12] H. T. Madhloom, S. A. Kareem, and H. Ariffin, "Computer-aided acute leukemia blast cells segmentation in peripheral blood images," Journal of Vibroengineering, vol. 17, no. 8, pp. 4517-4532, 2015.

[13] M. Barani, M. Mukhtar, A. Rahdar, G. Sargazi, A. Thysiadou, and G. Z. Kyzas, "Progress in the Application of Nanoparticles and Graphene as Drug Carriers and on the Diagnosis of Brain Infections," Molecules, vol. 26, no. 1, p. 186, 2021.

[14] J. E. Greenlee and K. C. Carroll, "Cerebrospinal fluid in central nervous system infections," Infections of the central nervous system, vol. 3, p. 5, 2004.

[15] D. Sánchez-Ruiz, I. Olmos-Pineda, and J. A. Olvera-López, "Automatic region of interest segmentation for breast thermogram image classification," Pattern Recognition Letters, vol. 135, pp. 72-81, 2020.

[16] Z. AKÇA, "High Performance Automated Diagnosis System for Hypertensive Retinopathy," Ankara Yıldırım Beyazıt Üniversitesi Fen Bilimleri Enstitüsü, 2017.

[17] N. K. Natt, H. Kaur, and G. Raghava, "Prediction of transmembrane regions of $\beta$-barrel proteins using ANN-and SVM-based methods," PROTEINS: Structure, Function, and Bioinformatics, vol. 56, no. 1, pp. 11-18, 2004.

[18] C. Deneke, R. Rentzsch, and B. Y. Renard, "PaPrBaG: A machine learning approach for the detection of novel pathogens from NGS data," Scientific reports, vol. 7, no. 1, pp. 1-13, 2017. 
[19] B. Pradhan and S. Lee, "Landslide susceptibility assessment and factor effect analysis: backpropagation artificial neural networks and their comparison with frequency ratio and bivariate logistic regression modelling," Environmental Modelling \& Software, vol. 25, no. 6, pp. 747-759, 2010.

[20] N. Wang, Y. Zhang, Z. Li, Y. Fu, W. Liu, and Y.-G. Jiang, "Pixel2mesh: Generating 3d mesh models from single rgb images," in Proceedings of the European Conference on Computer Vision (ECCV), 2018, pp. 52-67.

[21] S. S. Chouhan, A. Kaul, and U. P. Singh, "Image segmentation using computational intelligence techniques," Archives of Computational Methods in Engineering, vol. 26, no. 3, pp. 533-596, 2019.

\section{Author's Short Biography}

\begin{tabular}{|c|c|}
\hline & $\begin{array}{l}\text { In } 2021 \text { Kalthom Ibrahim received her PhD in Electrical control Engineering (University of Al } \\
\text { neelain). She Obtained international PCT under publication No WO 2019/147171 A2, report } \\
\text { generated on: } 03 \text { June } 2020 \text { (03.06.2020). Indian publication No. } 202017031635 \mathrm{~A} \text { (under process). } \\
\text { USA Patent Customer No: } 44696 \text { (under process). Her research interest includes control engineering } \\
\text { and micro processing,automation, electronic engineering }\end{array}$ \\
\hline & $\begin{array}{l}2009 \text { Mohammed Elmaleeh received his PhD degree in Electrical and Electronics Engineering } \\
\text { (University Technologist PETRONAS, Malaysia). Since then, Elmaleeh published numerous papers } \\
\text { in the field of Electronics Engineering. He supervised many postgraduate and undergraduate } \\
\text { students. He was assigned as a reviewer for several IEEE conferences and international journals. } \\
\text { Elmaleeh also was assigned as control engineering track chair for several IEEE conferences. His } \\
\text { research interest includes embedded system, electronic engineering, communication and computer } \\
\text { engineering }\end{array}$ \\
\hline & $\begin{array}{l}\text { Moaawia Ahmed have published much research. He continues his work in developing more } \\
\text { research, supervised above } 40 \text { master and PhD degrees dissertations spread on many universities } \\
\text { in Sudan and other regional countries. Research interest includes the field of control, automation, } \\
\text { and instrumentation. }\end{array}$ \\
\hline
\end{tabular}

